\title{
Laser Doppler flowmetry to assess myocardial microcirculation
}

\author{
Jakub Piotrowski ${ }^{1}$, Lech Anisimowicz ${ }^{1}$, Marcin Hellmann ${ }^{2}$ \\ ${ }^{1}$ Department of Cardiac Surgery, University Hospital, Bydgoszcz, Poland \\ ${ }^{2}$ Department of Cardiac Diagnostics, Medical University, Gdansk, Poland
}

\section{Introduction}

Several different methods enable the study peripheral microcirculation. Among them, non-invasive laser Doppler flowmetry (LDF) is routinely used to assess microvascular function by quantifying rapid changes in skin blood flow in response to a given pharmacological or mechanical stimulus [1]. Owing to its accessibility, peripheral microvascular function has been proposed as a diagnostic and prognostic marker in cardiovascular disease. Importantly, it has been shown that peripheral microvascular endothelial dysfunction correlates with endothelial function in coronary microvessels suggesting that endothelial dysfunction is a globalized pathological condition [2]. However, there is still a strong need in clinical practice to develop a tool which enables heart microcirculation in vivo assessment. Although, a direct assessment of the target microvascular bed such as coronary microcirculation is technically challenging.

Cardiac surgery, especially coronary artery bypass grafting (CABG), may be associated with several cardiovascular complications, including ischemia or acute myocardial infarction, arrhythmias and hemodynamic instability. Therefore, the possibility of intra- and postoperative myocardial microvascular perfusion assessment is of major clinical importance. Various studies have indicated that LDF is able to detect the real-time oscillations in myocardial perfusion in different experimental setups [3]. Of note, there is relatively little data concerning myocardial microvascular measurements in humans.

\section{Laser Doppler flowmetry mesurements}

The LDF technique is based on the emission of a beam of laser light carried by a fiber-optic probe. Tissue is illuminated with coherent laser light of $780 \mathrm{~nm}$ from a laser diode through a fiber optic light guide. The light undergoes changes in wavelength (Doppler shift) when it hits moving blood cells. The magnitude and frequency distribution of these changes in wavelength are related to the number and velocity of blood cells. Single point LDF assesses blood flow over a small volume (approximately $1 \mathrm{~mm}^{3}$ ) and is accurate at detecting and quantifying rapid changes in microvascular perfusion. Integrating probes made of several collecting optical fibers improve spatial resolution and the reproducibility of the technique. Of note, this technique does not provide an exact measure of flow ( $\mathrm{mL} / \mathrm{min})$. Indeed, LDF measurements are expressed as arbitrary perfusion units (PU) or as vascular conductance, which is flux divided by mean arterial pressure. Such an approach is more physiological as it takes into account differences and variations in blood pressure [1]. Importantly, all LDF measurements could be carried out continuously during surgery Figure $1 \mathrm{~A}$ presents a significant decrease of the myocardial microcirculation after artery occlusion during CABG, while on Figure 1B microcirculation remains constant during the same procedure in another patient. Of note, there are few papers regarding LDF perfusion measurements on the beating heart and most of them concern experimental animal studies [4].

Address for correspondence: Marcin Hellmann, MD, PhD, Department of Cardiac Diagnostics, Medical University, ul. Smoluchowskiego 17, 80-214 Gdańsk, Poland, tel: +48 5834933 80, fax: +48 5834933 79, e-mail: marcin.hellmann@gmail.com

Received: 16.12.2019 Accepted: 16.02.2020 


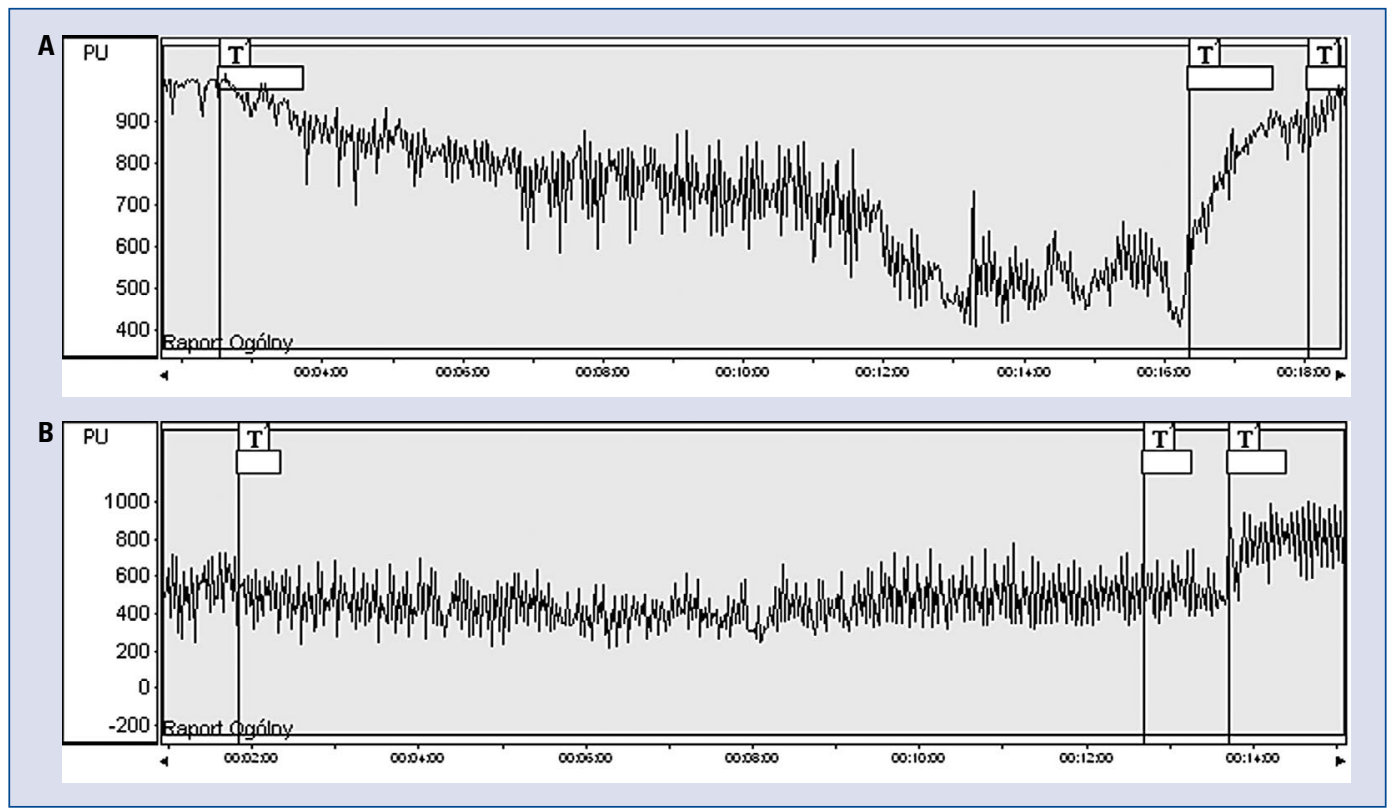

Figure 1. A. Significant decrease of the myocardial microcirculation after artery occlusion during coronary artery bypass grafting (CABG); B. Microcirculation remains constant after artery occlusion during CABG.

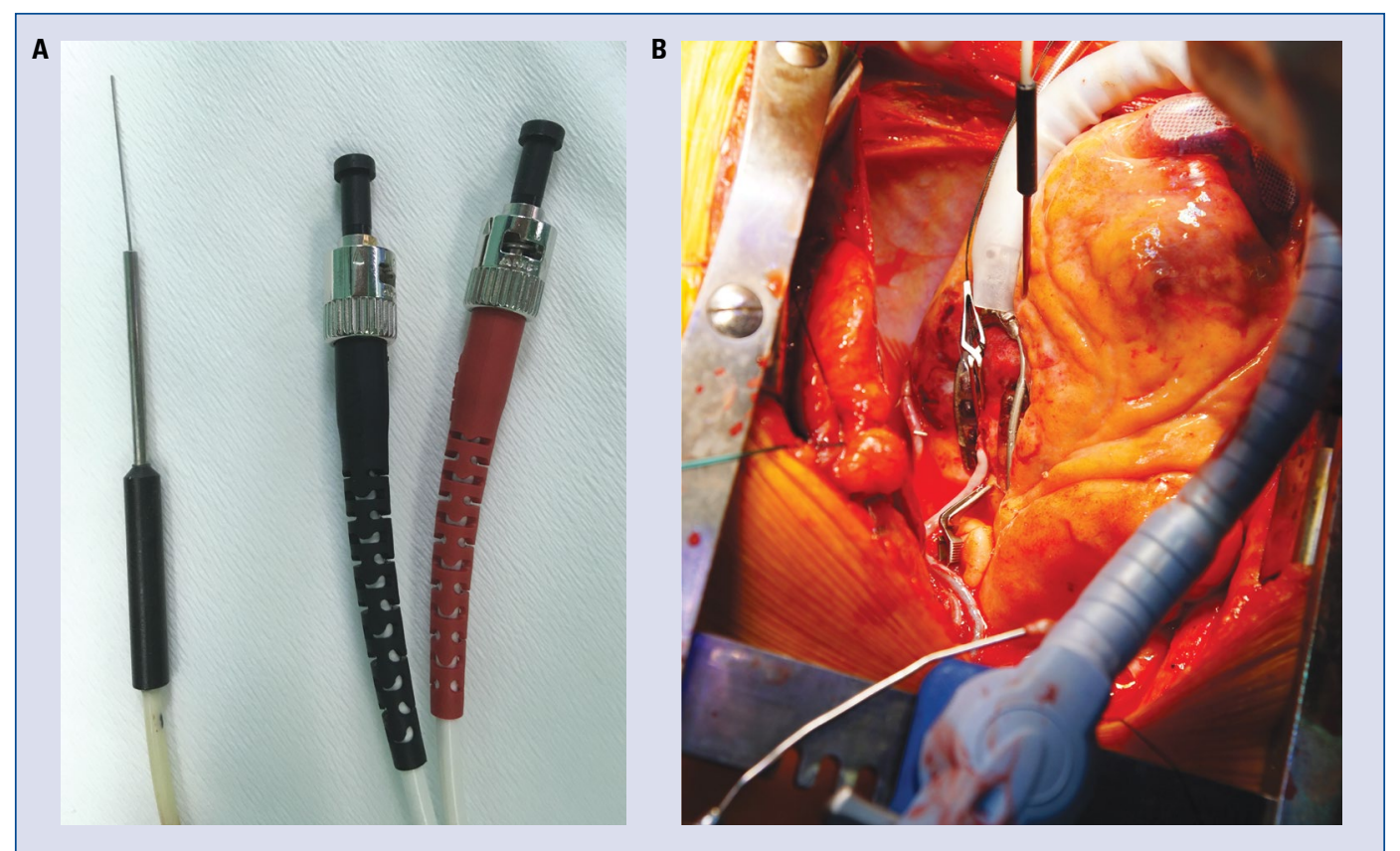

Figure 2. A. Laser Doppler flowmetry intramuscular perfusion probe; B. Laser Doppler flowmetry intramuscular perfusion probe was inserted into the myocardium during coronary artery bypass grafting downstream from the planned anastomosis.

A recently published case report presenting a patient with extensive coronary artery disease undergoing off-pump CABG. All measurements of myocardial microvascular perfusion were carried out using commercially available LDF (Periflux
System 5000, Perimed, Järfälla, Sweden) equipped with a special insertion probe (Stainless Steel Probe 411-311, Perimed, Järfälla, Sweden). Laser Doppler intramuscular fiber-optic perfusion probe (Fig. 2A) was inserted 3-5 $\mathrm{mm}$ into the myocar- 
dium during coronary surgery downstream from the planned anastomosis (Fig. 2B). It was shown that myocardial microcirculation remained constant after coronary artery occlusion and during performing the anastomosis [5]. It was hypothesized that it could be due to a well-developed coronary collateral circulation which was well-illustrated on the angiogram [6].

A currently running pilot study in patients undergoing $\mathrm{CABG}$ procedures is underway. Preliminary observations showed that insertion of the laser Doppler perfusion probe into the myocardium during cardiac surgery was safe and well-tolerated. Although invasive, this procedure was not associated with any cardiac side effects. Of note, laser Doppler perfusion measurements are reproducible and highly sensitive to acute changes in myocardial microvascular perfusion. According to available research, only LDF with intramuscular fiber-optic perfusion probe enables direct assessment of endocardial microcirculation. Newly-developed laser speckle contrast imaging provides an index of microvascular blood flow. Laser speckle contrast imaging allows non-contact and non-invasive real-time monitoring of microcirculatory perfusion on a wide area of tissue with a very good spatial resolution and an excellent reproducibility [1]. However, it could only measure a superficial layer of the tissue. Therefore, it would be useful to exclusively assess epicardial blood flow.

\section{Conclusions}

The assessment of the myocardial microvascular perfusion is of essential interest in various experimental and clinical studies. Accumulating evidence suggests that coronary collateral circulation may protect against myocardial ischemia and other secondary cardiac complications. Laser Doppler perfusion monitoring would be useful in predicting myocardial ischemia during cardiac surgery.

\section{Acknowledgements}

Professor Marcin Hellmann received a scientific scholarship from the Polish Ministry of Science for the years 2016-2019.

\section{Conflict of interest: None declared}

\section{References}

1. Hellmann M, Roustit M, Cracowski JL. Skin microvascular endothelial function as a biomarker in cardiovascular diseases? Pharmacol Rep. 2015; 67(4): 803-810, doi: 10.1016/j. pharep.2015.05.008, indexed in Pubmed: 26321284.

2. Bonetti PO, Pumper GM, Higano ST, et al. Noninvasive identification of patients with early coronary atherosclerosis by assessment of digital reactive hyperemia. J Am Coll Cardiol. 2004; 44(11): 2137-2141, doi: 10.1016/j.jacc.2004.08.062, indexed in Pubmed: 15582310.

3. Cracowski JL, Roustit M. Current methods to assess human cutaneous blood flow: an updated focus on laser-based-techniques. Microcirculation. 2016; 23(5): 337-344, doi: 10.1111/micc.12257, indexed in Pubmed: 26607042.

4. Bierbach B, Scheewe J, Derfuss T, et al. Continuous regional myocardial blood flow measurement: validation of a near-infrared laser Doppler device in a porcine model. Microcirculation. 2012; 19(6): 485-493, doi: 10.1111/j.1549-8719.2012.00173.x, indexed in Pubmed: 22372528.

5. Hellmann M, Piotrowski J, Anisimowicz L, et al. A mystery of the myocardial microcirculation during coronary artery bypass grafting. Eur J Cardiothorac Surg. 2018; 54(2): 405, doi: 10.1093/ ejcts/ezy107, indexed in Pubmed: 29509883.

6. Seiler C. Assessment and impact of the human coronary collateral circulation on myocardial ischemia and outcome. Circ Cardiovasc Interv. 2013; 6(6): 719-728, doi: 10.1161/CIRCINTERVENTIONS.113.000555, indexed in Pubmed: 24347657. 